\title{
Spectroscopic phase microscopy for quantifying hemoglobin concentrations in intact red blood cells
}

\author{
YongKeun Park ${ }^{1,2}$, Toyohiko Yamauchi ${ }^{1,3}$, Wonshik Choi ${ }^{1,4}{ }^{*}{ }^{*}$, Ramachandra Dasari ${ }^{1}$, and \\ Michael S. Feld ${ }^{1}$ \\ ${ }^{1}$ George R. Harrison Spectroscopy Laboratory, MIT, 77 Massachusetts Avenue, Cambridge, MA \\ 02139, USA \\ 2 Department of Health Science \& Technology, Harvard-MIT, 77 Massachusetts Avenue, Cambridge \\ MA 02139, USA \\ ${ }^{3}$ Hamamatsu Photonics K. K., 5000 Hirakuchi, Hamakita, Shizuoka 434-8601 Japan \\ 4 Department of Physics, Korea University, Seoul 136-701, Korea
}

\begin{abstract}
We report a practical method for label-free quantification of specific molecules using spectroscopic imaging of sample-induced phase shifts. Diffraction phase microscopy equipped with various wavelengths of light source is used to record wavelength-dependent phase images. We first perform dispersion measurements on pure solutions of single molecular species present in the cells, such as albumin and hemoglobin $(\mathrm{Hb})$. With this prior calibration of molecular specific dispersion, we demonstrate the extraction of $\mathrm{Hb}$ concentration from individual red blood cells (RBCs). The end point of this study is non-invasive monitoring of physiological states of intact living cells.
\end{abstract}

Biochemical activity of molecules governs cellular metabolism, which makes molecular concentrations important indicators of patho-physiological states of cells or tissues. Staining agents such as fluorescent dye and fluorescent protein have been widely used to quantify molecules of interest. However, the quantification of molecules using such techniques requires careful consideration on the artifacts of the staining agents: photo-bleaching, interference with metabolic activities of living cells, and non-uniform binding to the target molecules.

Refractive index (RI) can provide molecular concentration without such artifacts. As is well known, RI is related to the concentration of cellular dry mass [1]. Quantitative phase microscopy (QPM) has proven to be a useful tool to measure the phase delay induced by a specimen, which is related to its RI [2-8]. However, two limitations prevent it from retrieving specific molecular concentrations from the phase delay measurements. First, the measured phase delay implies approximate total protein contents, not the concentration [1,9]. The phase delay is an integral RI in the cell volume, and the RI should be decoupled from thickness to obtain molecular concentration. Second, both the phase and RI are not specific enough to differentiate single molecular species from mixtures. All types of molecules present in the cell can contribute to the RI. We note that the wavelength-dependent RI, obtained via dispersion, can resolve both limitations simultaneously. Many types of molecules can be differentiated by their dispersion. For example, $\mathrm{Hb}$ has significant dispersion in the visible wavelengths.

*Corresponding author: wonshik@mit.edu.

OCIS codes: (180.0180) Microscopy; (120.0120) Instrumentation, measurement, and metrology; (170.0170) Medical optics and biotechnology 
Dispersion was previously used to decouple RI from thickness of cells from the dispersion of a fluorescent medium [7], and to measure the concentration of molecules in solution and in tissue sections [10].

This letter presents a new technique, spectroscopic phase microscopy (SPM), which can measure the concentration of specific molecules in living cells and their volumes at the same time. SPM integrates diffraction phase microscopy (DPM) $[5,11]$ and a white-light source with various bandpass filters to select various wavelengths. We demonstrate the simultaneous extraction of $\mathrm{Hb}$ concentration and cell volume of intact individual RBCs. Abnormalities in $\mathrm{Hb}$ concentration are associated with various $\mathrm{RBC}$ diseases (sickle-cell anemia, thalassemia, and malaria infection) [6], and the present technique will potentially lead to assessing these disease conditions quantitatively.

The experimental setup consists of two parts: a white-light source with color filters for wavelength selection, and DPM for measuring quantitative phase delay (Fig. 1). DPM provides highly stable phase measurements, while the use of color filters simplifies the experimental setup in switching wavelength of the light source. In dispersion measurements, a wide range of wavelengths is necessary. Either a tunable laser or a set of different lasers can be used, either of which is costly. Instead, we used a Xenon arc lamp with a set of bandpass filters. Seven different filters are used to select various center wavelengths: $440 \pm 20 \mathrm{~nm}, 546 \pm 10 \mathrm{~nm}, 560$ $\pm 20 \mathrm{~nm}, 580 \pm 25 \mathrm{~nm}, 600 \pm 20 \mathrm{~nm}, 655 \pm 20 \mathrm{~nm}$, and $700 \pm 20 \mathrm{~nm}$. The common-path geometry of DPM matches the optical path lengths for the sample and reference arms such that the alignment is independent of the wavelength and temporal coherence of the illumination source. However, it still requires long spatial coherence, due to a spatial filtering process to be described. Thus, the size of aperture stop was minimized to increase spatial coherence. A specimen, located at the sample stage of an inverted microscope, is projected to the image plane IP1. The image was further magnified and delivered to the IP2, where a holographic grating was placed to generate multiple diffraction orders. The $0^{\text {th }}$ and $1^{\text {st }}$ order beams were isolated. The $0^{\text {th }}$ order beam is spatially low-pass filtered using a pinhole in a 4-f lens system; the beam then became a clean plane wave at the camera plane. The $1^{\text {st }}$ order beam served as sample beam. Both beams interfered and generated a spatially modulated interference image, which was then captured by a CCD camera. Since both beams share almost the same beam path, common-mode phase noise was cancelled out upon interference. The E-field was extracted from the recorded interferogram by spatial Hilbert transform [5]. The grating period, $30 \mu \mathrm{m}$, was set to be smaller than the diffraction-limited spot of the microscopic imaging system at the grating plane. All the lenses were achromatic to minimize chromatic dispersion.

To assess the ability of SPM, we measured the RIs of polydimethylsiloxane (PDMS), Bovine serum albumin (BSA), and $\mathrm{Hb}$ solutions at various wavelengths. Since the phase image presents relative phase delay between a sample and a medium, phase reference is required to determine absolute phase change induced by a specimen. We used a micro-fluidic channel and the RI of water for this purpose. We filled the PDMS channel with distilled water, measured quantitative phase images at different wavelengths. The relative phase delay between PDMS and water is given by $\Delta \phi=2 \pi / \lambda\left(n_{P D M S}(\lambda)-n_{w}(\lambda)\right) h$, where $\lambda, h, n_{P D M S}$ and $n_{w}$ are the wavelength of the light, channel height, RI of PDMS, and RI of water, respectively. Given the known dispersion of water [12] and $h=10 \mu \mathrm{m}$, we obtained $n_{P D M S}$ (Fig. 2g). Next, we measured the RI of the BSA solution. The measured dispersion of PDMS served as reference this time. The PDMS channel was filled with BSA solution $(300 \mathrm{mg} / \mathrm{ml}$; Gibco). From the relative phase to PDMS, the dispersion of BSA solutions were acquired using the relation, $\Delta \phi=2 \pi\left(n_{P D M S}(\lambda)-n_{B S A}\right.$ $(\lambda ; C)) h / \lambda$, with $n_{B S A}(\lambda ; C)$ the RI of BSA solution at wavelength $\lambda$ and concentration $C$. The obtained $n_{B S A}$ (Fig. 2g) is consistent with the literature [13]. Next, we measured the dispersion of $\mathrm{Hb}$ solutions, prepared from $\mathrm{Hb}$ protein powder (H7379, Sigma-Aldrich, Inc.), at three different concentrations: $0.05,0.15$, and $0.30 \mathrm{~g} / \mathrm{ml}$, respectively (Figs 2a-f). Using the method 
above, we obtained $n_{H b}(\lambda ; C)$ (Fig. 2h), which is consistent with that of the literature [14]. From this measurement, we retrieved a linear relationship between the RI of $\mathrm{Hb}$ solution and its concentration at various wavelengths [1]: $n_{H b}(\lambda ; C)=\alpha(\lambda) C+n_{w}$, with $\alpha(\lambda)$ a specific RI increment for $\mathrm{Hb}: \alpha(440)=0.240 \pm 0.007 \mathrm{ml} / \mathrm{g}, \alpha(560)=0.227 \pm 0.004 \mathrm{ml} / \mathrm{g}$, and $\alpha(660)=$ $0.221 \pm 0.005 \mathrm{ml} / \mathrm{g}$. The albumin protein in BSA solution does not show significant dispersion in the visible range (inset, Fig. $2 \mathrm{~g}$ ); $\alpha(\lambda)$ is $0.183 \pm 0.003 \mathrm{ml} / \mathrm{g}$ for the wavelength range of 440 $-700 \mathrm{~nm}$.

Next, using the calibrated dispersion of Hb solution we applied SPM to measure cytoplasmic $\mathrm{Hb}$ concentration in live human RBCs. Fresh blood $(5 \mathrm{ml})$ obtained by venipuncture were diluted in Phosphate-Buffered Saline (PBS) solution, and then washed three times to remove white blood cells and platelets. The interference images of RBCs were measured at the seven wavelengths (Figs. 3a-c). The phase images of a RBC can be expressed as, $\Delta \phi(x, y ; \lambda)=2 \pi$ $\left(\alpha(\lambda) C(x, y)+n_{X}(x, y, \lambda)\right) h(x, y) / \lambda$, with $h(x, y)$ the local thickness of the cell and $n_{X}(x, y, \lambda)$ an relative averaged RI of other molecules besides $\mathrm{Hb}$ in $\mathrm{RBC}$ compared to water (non- $\mathrm{Hb}$ proteins ( $2.5 \%$ of total protein mass) and ionic molecules in cytosolic space [15]). These non$\mathrm{Hb}$ molecules usually do not have distinct dispersion at visible wavelengths. For example, albumin, most abundant plasma protein in mammals, has almost the same trend in RI as water in the wavelength range $440-700 \mathrm{~nm}$ (inset, Fig. $2 \mathrm{~g}$ ). Thus, we can approximate $n_{X}(x, y, \lambda)$ as an independent constant of wavelength, $n_{X}$. Since $\alpha(\lambda)$ was calibrated above, there are three unknowns: $C, n_{X}$, and $h(x, y)$. Thus, the phase measurements at three different wavelengths are sufficient to extract $C, n_{X}$, and $h(x, y)$ simultaneously. In principle, we can distinguish $N$ different molecular contents with $N+2$ spectroscopic phase measurements, assuming that they have distinctive dispersion from one another. Fig. 3d shows the retrieved $\mathrm{Hb}$ concentration, $C$ $(x, y)$ of a RBC. Fig. 3e is a histogram of the $\mathrm{Hb}$ concentration in $\mathrm{RBCs}(\mathrm{N}=25)$. The average value is $0.318 \pm 1.7 \mathrm{~g} / \mathrm{ml}$. From the measured area of the cells and corresponding thickness, we calculated the cell volume (Fig. 3f). The average value is $90.5 \pm 3.3 \mathrm{fl}$. These results are within normal physiological range [15]. According to the measured conversion factors, the sensitivity of SPM is $0.0009-0.0014 \mathrm{~g} / \mathrm{ml}$ for $\mathrm{Hb}$ concentration, since the sensitivity in phase measurement of DPM is $\sim 3 \mathrm{mrad}$ [11]. This sensitivity corresponds to $0.08-0.13 \mathrm{pl}$ for $\mathrm{Hb}$ contents per cell, assuming the average red blood cell volume is $90 \mathrm{fl}$.

In summary, we have presented SPM, a simple and practical method for spectroscopic phase imaging, which can simultaneously measure $\mathrm{Hb}$ concentrations and cell volume of living RBCs. This new instrument has many potential applications, of one we give here; quantifying isomeric shifts in $\mathrm{Hb}$ (oxy- and deoxy-Hb) when exposed to different oxygen pressures. Binding of oxygen to $\mathrm{Hb}$ proteins cause conformational changes which result in significant differences in dispersion. Thus, SPM instrument may provide a valuable tool for better understanding of oxygen transport by RBCs.

\section{Acknowledgments}

This work was funded by the National Center for Research Resources of the National Institutes of Health (P41RR02594-18), the National Science Foundation (DBI-0754339) and Hamamatsu Corporation. Y.-K. Park was supported by Samsung Fellowship.

\section{References}

1. Barer R. Determination of dry mass, thickness, solid and water concentration in living cells. Nature 1953;172:1097-1098. [PubMed: 13111263]

2. Cuche E, Bevilacqua F, Depeursinge C. Digital holography for quantitative phase-contrast imaging. Opt Lett 1999;24:291-293. [PubMed: 18071483]

3. Barty A, Nugent K, Paganin D, Roberts A. Quantitative optical phase microscopy. Optics Letters 1998;23:817-819. [PubMed: 18087351] 
4. Rylander C, DDP, Akkin T, Milner T, Diller K, Welch A. Quantitative phase-contrast imaging of cells with phase-sensitive optical coherence microscopy. Optics Letters 2004;29:1509-1511. [PubMed: 15259729]

5. Popescu G, Ikeda T, Dasari RR, Feld MS. Diffraction phase microscopy for quantifying cell structure and dynamics. Opt Lett 2006;31:775-777. [PubMed: 16544620]

6. Park YK, Diez-Silva M, Popescu G, Lykotrafitis G, Choi W, Feld MS, Suresh S. Refractive index maps and membrane dynamics of human red blood cells parasitized by Plasmodium falciparum. Proc Natl Acad Sci USA 2008;105:13730. [PubMed: 18772382]

7. Rappaz B, Charriere F, Depeursinge C, Magistretti PJ, Marquet P. Simultaneous cell morphometry and refractive index measurement with dual-wavelength digital holographic microscopy and dyeenhanced dispersion of perfusion medium. Optics Letters 2008;33:744-746. [PubMed: 18382537]

8. Park Y, Choi W, Yaqoob Z, Dasari R, Badizadegan K, Feld M. Speckle-field digital holographic microscopy. Optics Express 2009;17:12285-12292. [PubMed: 19654630]

9. Popescu G, Park Y, Lue N, Best-Popescu C, Deflores L, Dasari R, Feld M, Badizadegan K. Optical imaging of cell mass and growth dynamics. Am J Physiol: Cell Physiol 2008;295:C538. [PubMed: 18562484]

10. Yang C, Wax A, Georgakoudi I, Hanlon E, Badizadegan K, Dasari R, Feld M. Interferometric phasedispersion microscopy. Optics Letters 2000;25:1526-1528. [PubMed: 18066267]

11. Park YK, Popescu G, Badizadegan K, Dasari RR, Feld MS. Diffraction phase and fluorescence microscopy. Opt Express 2006;14:8263-8268. [PubMed: 19529201]

12. Schiebener P, Straub J, Sengers J, Gallagher J. Refractive index of water and steam as function of wavelength, temperature and density. J Phys Chem Ref Data 1990;19:677-717.

13. Andersen M, Painter L, Nir S. Dispersion equation and polarizability of bovine serum albumin from measurements of refractive indices. Biopolymers 1974;13

14. Friebel M, Meinke M. Model function to calculate the refractive index of native hemoglobin in the wavelength range of 250-1100 nm dependent on concentration. Applied optics 2006;45:2838-2842. [PubMed: 16633438]

15. Lichtman, M. Williams hematology. McGraw-Hill Professional; 2005. 


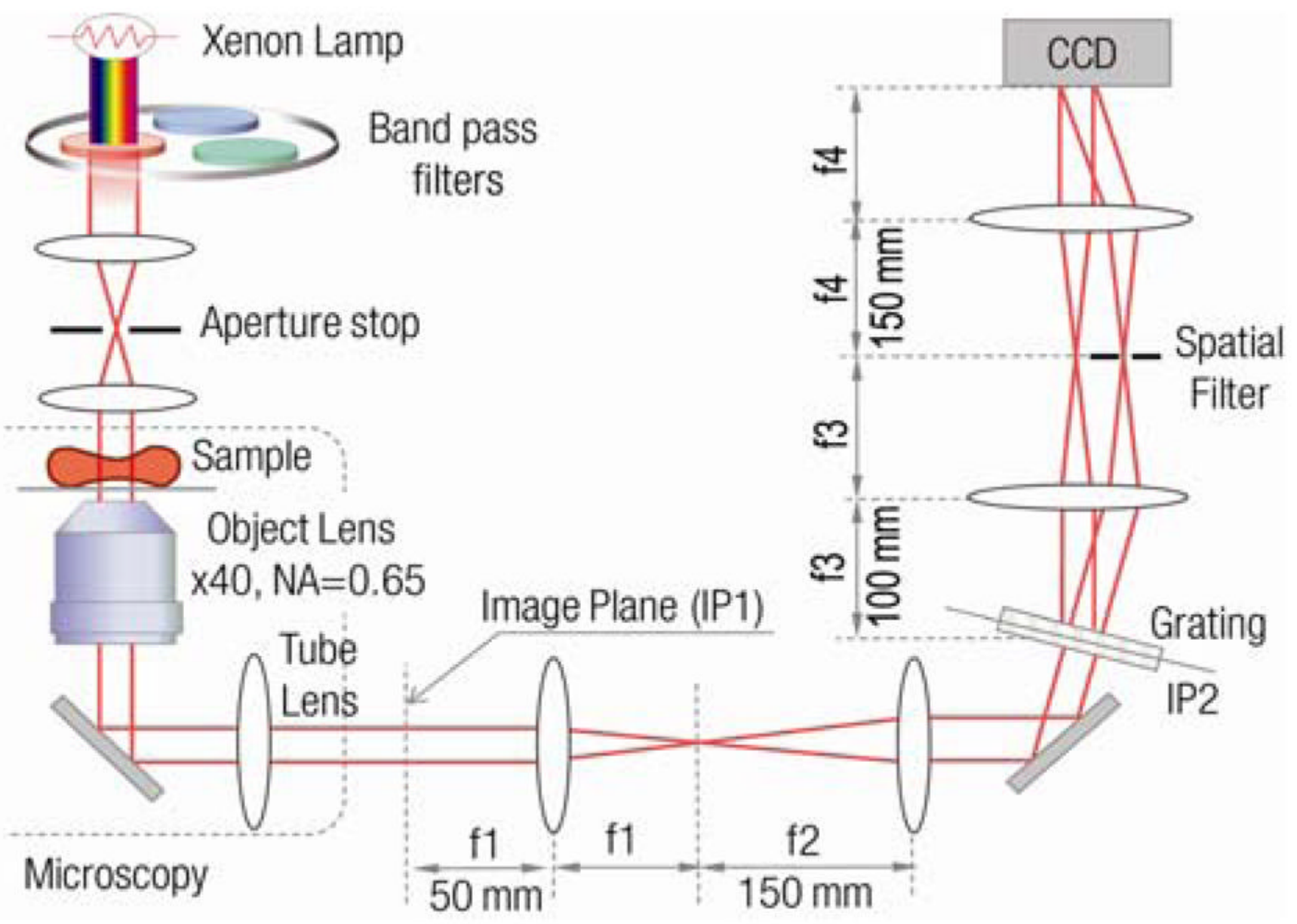

Fig. 1.

(Color online) Experimental setup. 

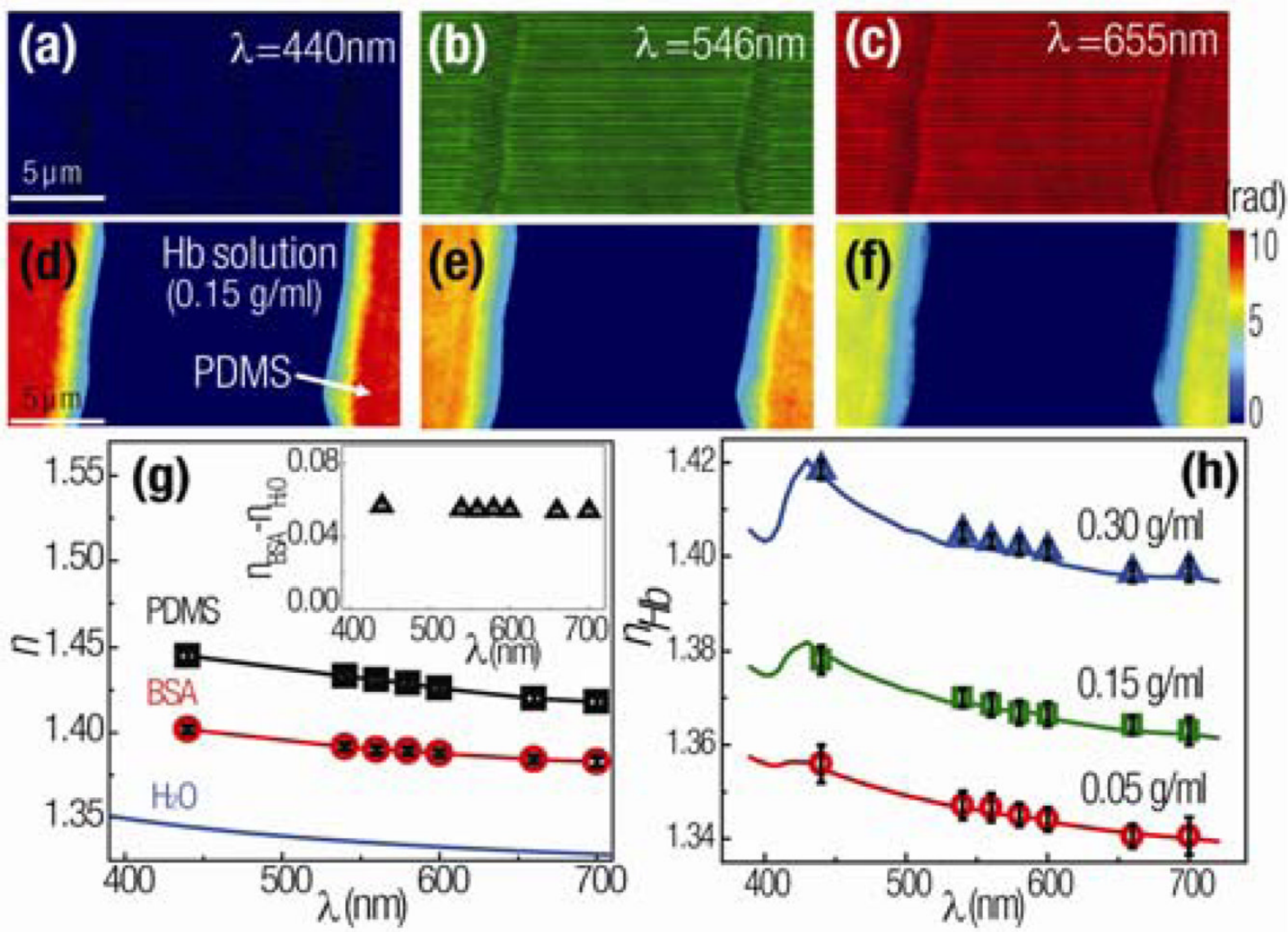

Fig. 2.

(Color online) (a-c) Interferograms of PDMS channel filled with Hb solution. (d-f) Corresponding quantitative phase maps (g) RIs of PDMS, BSA, and water. RIs of water are adopted from Ref. [12]. Inset, the RI difference between BSA solution and water. (h) Measured dispersion of $\mathrm{Hb}$ solutions (symbols) and comparison with Ref. [14] (lines). Error bars indicate standard deviations. 

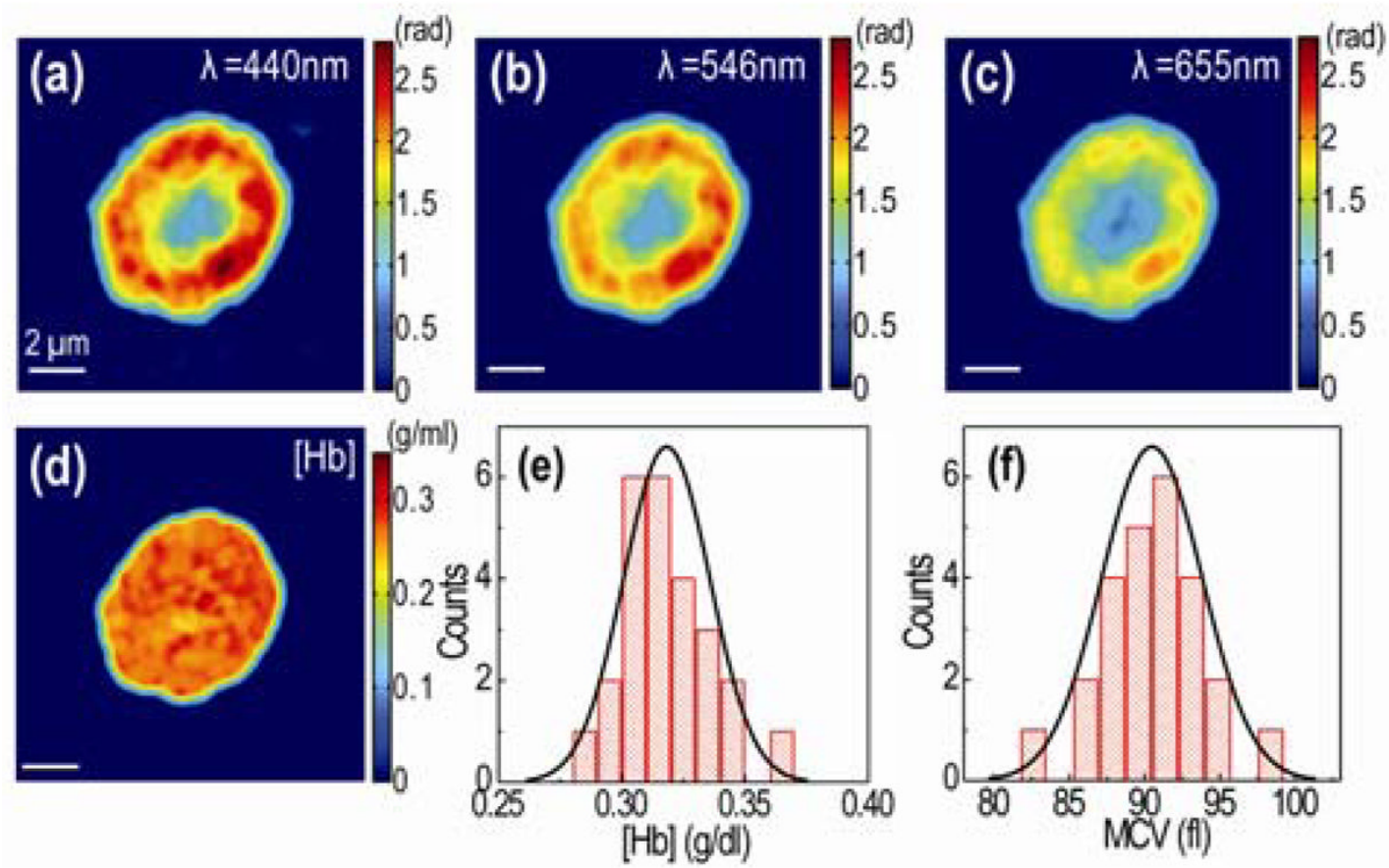

Fig. 3.

(Color online) (a-c) Quantitative phase maps of a RBC at three different wavelengths. (d) The retrieved $\mathrm{Hb}$ concentration. (e) Histogram of $\mathrm{Hb}$ concentrations and (f) cell volumes ( $\mathrm{N}=25)$. 